\title{
Analysis of Flow-Mediated Dilation by Nitric Oxide Production Model of Vascular Endothelial Cells
}

\author{
Akira Tsukamoto, Yoshimi Kamiyama \\ Information Science and Technology, Aichi Prefectural University \\ 1522-3, Ibaragabasama, Nagakute, Aichi 480-1198, Japan \\ tsukamoto@nil.ist.aichi-pu.ac.jp; kamiyama@ist.aichi-pu.ac.jp
}

\begin{abstract}
The vascular endothelial function is impaired in early stages of atherosclerosis. The flow-mediated dilation (FMD) test is the most common noninvasive method used to assess endothelial function. In this study, to analyze the endothelial function in detail using the FMD test, we developed a novel mathematical model that can simulate the FMD test. We used data assimilation with observation data of the mean blood flow velocity and estimated vessel property model parameters. We confirmed that the present model reproduces the observation data, and the estimated parameters are physiologically acceptable. The model is also applicable for the simulation of the dynamics of intracellular ion movements to analye the physiological mechanism of the endothelial function.
\end{abstract}

Keywords: Atherosclerosis, FMD, Endothelial Cells, Nitric Oxide, Mathematical Model

\section{Introduction}

The vascular endothelial function of endothelial cells plays a key role in maintaining normal vascular homeostasis. The vascular endothelial cells constituting the tunica intima produce vasoactive substances such as nitric oxide (NO), which could cause the relaxation of vascular smooth muscle and the inhibition of vessel wall inflammation. Because the vascular endothelial function is impaired in early stages of atherosclerosis, it is possible to treat the disease early by detecting endothelial dysfunction [1]. Therefore, it is important to evaluate vascular endothelial function as an index of atherosclerosis.

The flow-mediated dilation (FMD) test is the most common noninvasive method to assess endothelial function. Because it is difficult to measure NO directly, the endothelial function is evaluated by measuring endothelial-dependent vasodilation in the upper arteries. Vasodilation (FMD response) is measured before and after an increase in shear stress, which is induced by reactive hyperemia. However, because the physiological mechanism of vasodilation is highly complicated, the details of endothelial function are unclear in the test.

Mathematical models are helpful to adequately understand the mechanisms of NO production. However, because most mathematical models for NO production solely describe individual physiological pathways, it is difficult to analyze the endothelial function in its entirety. In a previous study, we developed a mathematical model to analyze NO production [2]. This model simulates intracellular reactions during NO production induced by shear stress. In the present study, we propose an improved model that integrates hemodynamics and NO production. In the previous multi-branched arterial model proposed by Avolio, hemodynamics and vasomotor responses could be simulated in humans [3]. However, the model is not applicable for simulating dynamic phenomena, because the vascular wall is expressed as an elastic tube with constant parameters. To resolve this problem, we introduced a data assimilation method to estimate the parameters and reproduce reactive hyperemia. We also simulated the dynamics of intracellular ion movement to analyze the physiological mechanism and sequence of endothelial function.

\section{Material and Method}

\subsection{Physiological Mechanisms of FMD Response}

The FMD test is a noninvasive method used to assess the endothelial function. The technique causes the release of NO, resulting in vasodilation, and measures continuous changes in arterial diameter and blood flow throughout the test, described as follows [4]. A cuff is placed on the forearm and inflated. The vessel diameter of the upper arm is measured using an ultrasonic probe, by the following process (Fig.1 (a)).

1. Cuff inflation at the forearm $(5 \mathrm{~min})$ 
2. Sharp increase in blood flow velocity after cuff deflation (reactive hyperemia)

3. NO production from vascular endothelial cells

4. Increase in vessel diameter

Fig. 1 (b) shows sample results. FMD is determined by the magnitude of postischemic vasodilatation according to the following expression:

$$
F M D(\%)=\frac{D_{\text {max }}-D_{\text {rest }}}{D_{\text {rest }}} \times 100
$$

where $D_{\text {rest }}$ is the resting diameter and $D_{\max }$ is the maximum diameter. A principal factor of FMD is endothelium-derived NO. However, the precise mechanisms of shear stress and subsequent signal transduction are not fully understood. Shear stress is a key activator of endothelial NO synthase (eNOS) in normal physiology. NO production is regulated via the activation and expression of eNOS and accounts for FMD.

\subsection{Multi-Branched Arterial Model}

To investigate vessel properties during the FMD test, a multi-branched model proposed by Avolio was employed to simulate hemodynamic features. The model divides the artery into 128 segments of short elastic tubes. Each segment is represented by an equivalent two-input-two-output electrical circuit. Blood pressure $P$ and blood flow $Q$ are described as voltage $V$ and current $I$, respectively.

$$
\begin{aligned}
& -\frac{\delta P}{\delta x}=Q R+L \frac{\delta Q}{\delta t} \\
& -\frac{\delta Q}{\delta x}=P G-C \frac{\delta P}{\delta t}
\end{aligned}
$$

Here, $P$ is blood pressure, $Q$ is blood flow, $x$ is distance, and $t$ is time. Parameters R, I, C and G depend on both the rheological properties of blood and the geometrical characteristics of the vessel segment. These segments are connected based on anatomical knowledge and reproduce the characteristics of blood flow velocity and pressure.

All terminations to peripheral segments consist of a pure resistance determined by the reflection coefficient $\Gamma$. The peripheral impedance $Z_{L}$ is calculated as the arteriolar and lower arteries, and peripheral resistance $R_{L}$ is obtained as follows:

$$
\begin{gathered}
Z_{L}=\sqrt{\frac{R+j \omega L}{G+j \omega C}} \times \frac{1+\Gamma}{1-\Gamma} \\
R_{L}=\frac{1}{\operatorname{Re}\left(1 / Z_{L}\right)}
\end{gathered}
$$

Because peripheral resistance $R_{L}$ is constant, this model cannot reproduce dynamic changes such as FMD response. Reactive hyperemia is caused by a decrease in peripheral resistance, because of cuff inflation to produce ischemia. Thus, 

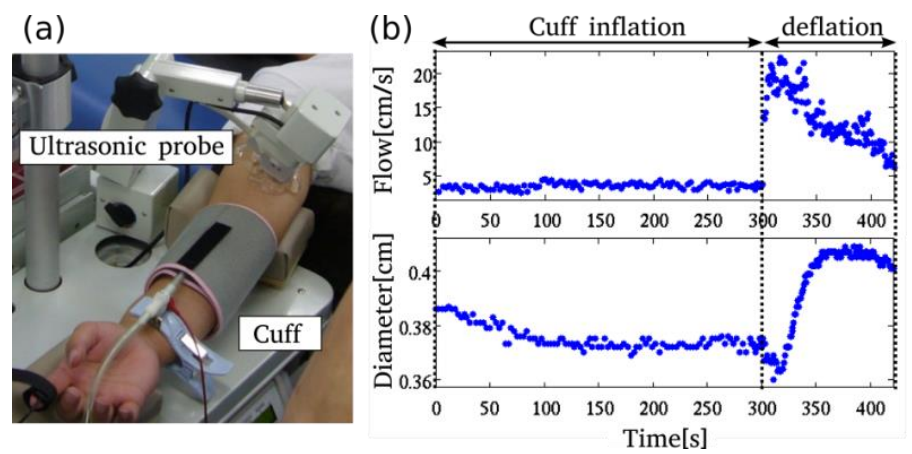

Fig. 1: FMD test (a) settings (b) and measured blood flow velocity and vessel diameter.

we need to estimate the dynamic characteristics of the peripheral site because FMD is a dynamic phenomenon. However, it is difficult to observe the dynamic changes of peripheral resistance.

We introduced the data assimilation method and estimated the peripheral resistance $R_{L}$ using the reflection coefficient Г. Fig.2 (b) shows a schematic description of the proposed model. The observation data is the mean blood flow velocity during the FMD test. We introduce the Kalman filter as the feedback law.

\subsection{Model of NO Production by Vascular Endothelial Cells}

We also use an endothelial NO production model based on physiological mechanisms to clarify the endothelial function. Ohashi proposed a multi-scale model of endothelial cells that simulates shear stress-induced intracellular ion movements and NO synthesis. The shear stress $\tau$ as the input stimulus for the endothelial NO production model is given by:

$$
\tau=\frac{4 \mu Q}{\pi r^{3}}
$$

where $\mu$ is blood viscosity and $r$ is vessel radius. The model is a combination of three types of models (physical, electrophysiological, and physiological models) developed based on physiological pathways.

The physical models calculate the deformation of endothelial cells by shear stress. Each component is regarded as a Kelvin body using the calculation method proposed by Mazzag [5]. The three components are integrin, membrane, and actin filaments.

The electrophysiological models simulate intracellular ion movement when the IP3 concentration in endothelial cell changes. The ion reaction is expressed by the conductance model proposed by Silva [6]. In addition, intracellular IP3 concentration is represented as a function of membrane deformation:

$$
\begin{gathered}
\frac{d[\mathrm{IP} 3]}{d t}=Q_{\mathrm{GIP} 3}-k_{\mathrm{DIP} 3}[\mathrm{IP} 3] \\
\frac{d Q_{\mathrm{GIP} 3}}{d t}=\frac{Q_{\mathrm{GIP} 3 \mathrm{SS}}\left(u_{\mathrm{Mem}}\right)-Q_{\mathrm{GIP} 3}}{\tau_{\mathrm{IP} 3}\left(u_{\mathrm{Mem}}\right)}
\end{gathered}
$$

where $k_{\mathrm{DIP} 3}$ is the rate constant of IP3 concentration, $Q_{\mathrm{GIP} 3}$ is the production rate of IP3, $Q_{\mathrm{GIP} 3 \mathrm{SS}}$ is the production rate of IP3 in steady state, and $\tau_{\text {IP3 }}$ is the time constant.

The physiological models are developed based on the model proposed by Koo [7]. The original model is not applicable for simulating NO production due to arbitrary stimuli during the FMD test. Therefore, we proposed an improved model to simulate NO concentration changes for different values of shear stress using inputs from physical and physiological models. 
(a)

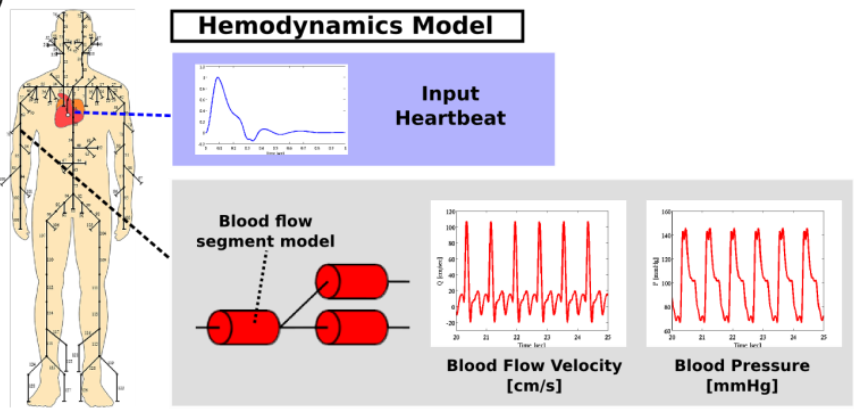

(b)

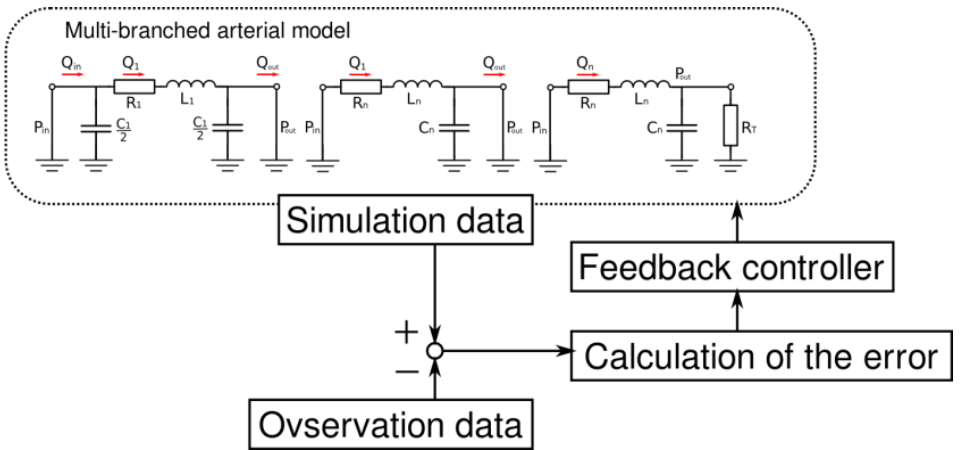

Fig. 2: (a) Structure of the hemodynamics model. (b) Schematic description using data assimilation.

\section{Results}

Fig. 3 shows the trends of mean blood flow velocity, shear stress, peripheral resistance, and NO concentration. The red dots denote the observation data points during the FMD test, and the blue lines denote the simulated results.

The simulation results reproduced the observation data and reactive hyperemia after cuff deflation well, i.e., blood flow velocity increased and peripheral resistance $\left(\mathrm{R}_{\mathrm{L}}\right)$ decreased. These estimated behaviors are physiologically acceptable. The proposed model also has the ability to simulate the dynamics of the intracellular ion movements from shear stress to NO production.
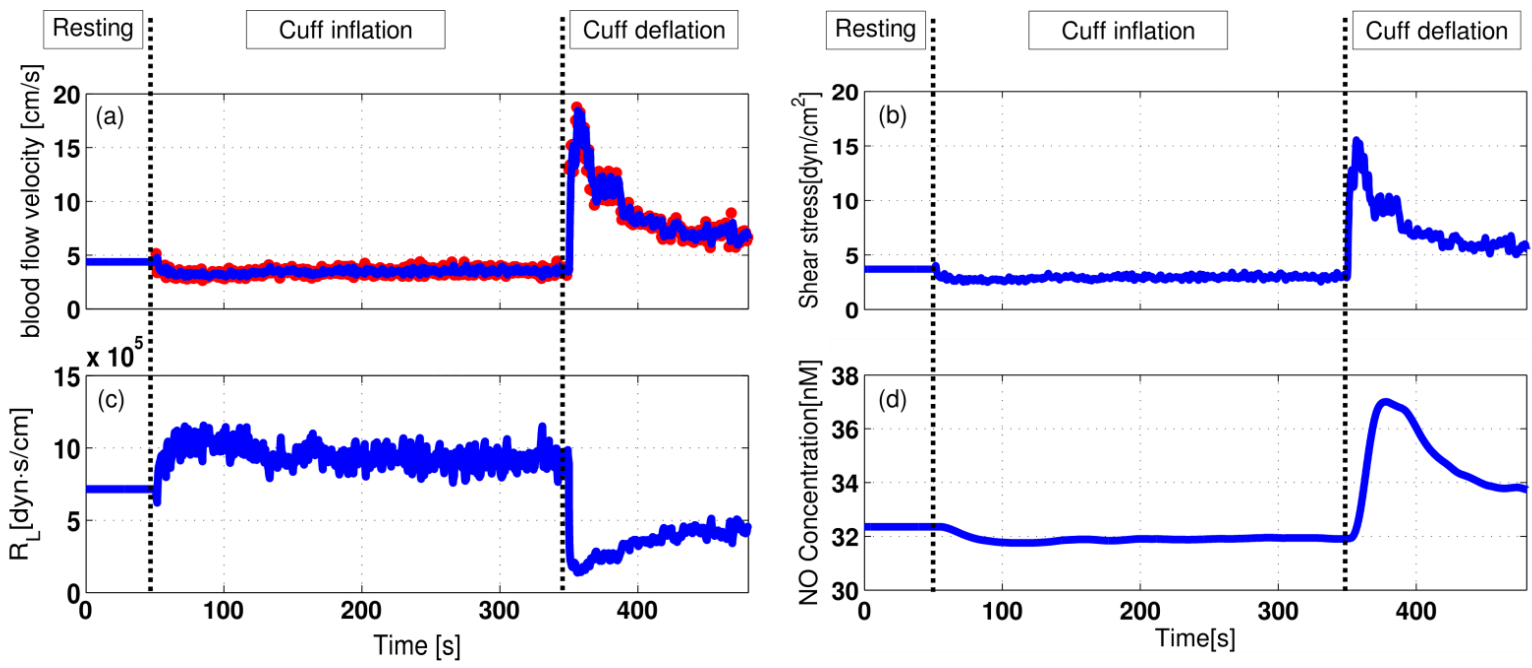

Fig. 3: Simulation results. The solid line represents the simulation result, and the red dots represent the observation data. (a) blood flow velocity, (b) shear stress, (c) reflection coefficient, (d) NO concentration.

\section{Conclusion}

We proposed an integrated mathematical model of the hemodynamic and NO production models. We also used data assimilation with observation data of the mean blood flow velocity and estimated the parameters of vessel property parameters. We found that the observation data and the estimated parameters obtained using the proposed model are physiologically acceptable. This model could prove to be a useful tool for analyzing physiological mechanism of endothelial function.

\section{Acknowledgements}

This study was supported by the Tokai Foundation and JSPS KAKENHI (17K00412). 


\section{References}

[1] R. Ross, "Atherosclerosis - an inflammatory disease," New England journal of medicine, vol. 340, no. 2, pp. 115-126, 1999.

[2] Y. Ohashi, et al., "Shear Stress-induced NO Production Model of Vascular Endothelial Cell," IEEJ Transactions on Electronics, vol. 136, no. 2, pp. 116-122, 2015.

[3] A. P. Avolio, "Multi-branched model of the human arterial system," Medical \& Biological Engineering \& Computing, vol. 18 , pp. 709-718, 1980 .

[4] M. C. Corretti, et al., "Guidelines for the ultrasound assessment of endothelial-dependent flow-mediated vasodilation of the human arterial system," Medical \& Biological Engineering \& Computing, vol. 42, pp. 312-321, 2002.

[5] B. M. Mazzg, et al., "A model for shear stress sensing and transmission in vascular endothelial cells," Biophysical Journal, vol. 84, pp. 4087-4101, 2003.

[6] H. S. Silva, et al., "A mathematical model of plasma membrane electrophysiology and calcium dynamics in vascular endothelial cells," AJP Cell Physiology, vol. 293, pp. 277-293, 2007.

[7] A. Koo, et al., "In silico modeling of shear-stress-induced nitric oxide production in endothelial cells through systems biology," Biophysical Journal, vol. 104, pp. 2295-2306, 2013. 